\title{
Article
}

\section{Cylindrical Gravitational Wave: Source and Resonance}

\author{
Yu-Zhu Chen ${ }^{1,2, *} \mathbb{0}$, Shi-Lin $\mathrm{Li}^{2}$, Yu-Jie Chen ${ }^{2}$ and Wu-Sheng Dai ${ }^{2, *}$ \\ 1 Theoretical Physics Division, Chern Institute of Mathematics and LPMC, Nankai University, \\ Tianjin 300071, China \\ 2 Department of Physics, Tianjin University, Tianjin 300350, China; lishilin@tju.edu.cn (S.-L.L.); \\ chenyujie@tju.edu.cn (Y.-J.C.) \\ * Correspondence: chenyuzhu@nankai.edu.cn (Y.-Z.C.); daiwusheng@tju.edu.cn (W.-S.D.)
}

Citation: Chen, Y.-Z.; Li, S.-L.; Chen, Y.-J.; Dai, W.-S. Cylindrical Gravitational Wave: Source and Resonance. Symmetry 2021, 13, 1425. https://doi.org/10.3390/ sym 13081425

Academic Editor: Piero Nicolini

Received: 5 July 2021

Accepted: 2 August 2021

Published: 4 August 2021

Publisher's Note: MDPI stays neutral with regard to jurisdictional claims in published maps and institutional affiliations.

Copyright: (c) 2021 by the authors. Licensee MDPI, Basel, Switzerland. This article is an open access article distributed under the terms and conditions of the Creative Commons Attribution (CC BY) license (https:/ / creativecommons.org/licenses/by/ $4.0 /)$.

\begin{abstract}
Gravitational waves are regarded as linear waves in the weak field approximation, which ignores the spacetime singularity. In this paper, we analyze singularities in exact gravitational wave solutions. We provide an exact general solution of the gravitational wave with cylindrical symmetry. The general solution includes some known cylindrical wave solutions as special cases. We show that there are two kinds of singularities in the cylindrical gravitational wave solution. The first kind of singularity corresponds to a singular source. The second kind of singularity corresponds to a resonance between different gravitational waves. When two gravitational waves coexist, the interference term in the source may vanish in the sense of time averaging.
\end{abstract}

Keywords: gravitational wave; singularity; source; resonance

\section{Introduction}

Singularity theorems [1] show that singularities are inevitable in spacetime while singularities are ignored in the linear approximation. For example, the conclusion that the dipole of the energy-momentum tensor vanishes in the center-of-mass coordinate [2] only makes sense in the absence of the singularity. In the linear approximation, we develop the gravitational wave scattering theory without large-distance asymptotics [3].

There are also research studies of gravitational waves with exact wave solutions of the Einstein equation. By the Birkhoff theorem, there is no gravitational wave in spherical vacuum spacetime $[2,4,5]$. The simplest spacetime related to gravitational waves is the cylindrical spacetime described by the Einstein-Rosen metric. The 1+3-dimensional cylindrical metric is similar to the 1+2-dimensional metric because of spacetime symmetry. Nevertheless, they are not the same. The 1+2-dimensional vacuum Einstein equation only has the Minkowski space solution such that gravitational waves do not exist in 1+2dimensional spacetime. The 1+3-dimensional cylindrical vacuum Einstein equation has infinite solutions. That is, the 1+3-dimensional cylindrical metric may contain gravitational wave solutions. A source of an infinite length may exist in the cylindrical spacetime so that a strictly cylindrical spacetime probably cannot exist. Nevertheless, it is convenient to discuss the theoretical problem about gravitational radiation with the cylindrical gravitational waves. In a series of works named "Gravitational waves in general relativity", various problems involving gravitational waves are discussed. In Reference [6], Marder provides static cylindrical solutions and superimposes cylindrical wave solutions on the static field. He also shows that cylindrical gravitational waves propagate energy. In Reference [7], the reflexion and the transmission of out-going cylindrical gravitational waves by a thin cylindrical shell of matter is discussed. In Reference [8], the gravitational field of a fast moving particle is discussed. In Reference [9-11], cylindrical gravitational waves are used to generate radiation solutions of other forms. There are many other works on gravitational waves taking advantage of cylindrical gravitational waves, such as the energy loss of the source by the gravitational radiation [12]; the C-energy, the super-energy, the associated 
dynamic effect of the cylindrical gravitational wave [13]; the energy-momentum of the gravitational wave [14]; the superenergy flux of the Einstein-Rosen wave [15]; the nonlinear effect such as the Faraday rotation and the time-shift phenomenon of the cylindrical gravitational solution [16]; the nonlinear evolution of cylindrical gravitational waves [17]; the twisted gravitational wave [18]; the scattering of the gravitational waves [19]; the gravitational collapse of the energy of gravitational waves [20]; the asymptotic structure of the radiation spacetime [21]; the interaction between the gravitational wave and the cosmic string [22,23]; the cosmic censorship hypothesis [24]; and the midisuperspace quantization [25-27].

In this paper, we discuss gravitational waves with exact cylindrical wave solutions. This paper is motivated by problems introduced in above paragraphs, which are the behavior of singularities in gravitational wave solutions and the new physical effects of gravitational wave solutions in addition to, e.g., the reflexion and the transmission. Based on the exact solution, we analyze singularities in gravitational waves. We show that there are two kinds of singularities in gravitational waves. The first kind of singularities lies at a fixed spatial position which corresponds to a source. We call it the source singularity. In Reference [6,7], cylindrical gravitational waves are considered as the exterior solutions of sources. We take a cylindrical gravitational wave as a complete solution. Our results shows that singularities in cylindrical gravitational waves carry the information about the source. The second kind of singularities arise as time proceeds to infinity. We recognize the singularity as a resonance and we call it the resonance singularity. In the literature, a resonance between gravitational radiation and the matter (especially the gravitational radiation detectors) is considered [28-31]. In our paper, however, it seems that a gravitational wave resonates with other gravitational waves. It is worth mentioning that the resonance singularity only emerges when a gravitational wave with a source singularity and a gravitational wave without a source singularity possess the same frequency. Two gravitational waves with source singularities or two gravitational waves without source singularities do not resonate. The resonance also indicates that the gravitational wave with sources and the gravitational wave without sources are two of different kinds.

General relativity is a nonlinear theory. The direct summation of two solutions are not a new solution any more. That results in a nontrivial superposition and interference of two gravitational waves. We show how the interference terms of two cylindrical gravitational waves behave. Interference terms arise both in the metric and the energy-momentum tensor. Specifically, we show that the interference term in the source vanishes in the sense of time averaging. To the best of our knowledge, the interference term in the source is rarely considered in other papers.

In Section 2, we provide a general cylindrical gravitational wave solution. In Section 3, we show the source singularity in a gravitational wave solution. In Section 4, we show the resonance between different gravitational waves. In Section 5 , we show the interference between two cylindrical gravitational waves. Conclusions and outlooks are provided in Section 6.

\section{Cylindrical Gravitational Wave: General Solution}

In this section, we provide a general solution of the cylindrical gravitational wave. Exact solutions are used to discuss the source of a gravitational wave solution and the resonance between gravitational waves. They are also used to discuss the interference of gravitational waves.

The cylindrical gravitational wave is described by the 1+3-dimensional Einstein-Rosen metric [32].

$$
d s^{2}=e^{2 \gamma(t, \rho)-2 \psi(t, \rho)}\left(-d t^{2}+d \rho^{2}\right)+e^{-2 \psi(t, \rho)} \rho^{2} d \phi^{2}+e^{2 \psi(t, \rho)} d z^{2} .
$$


The Einstein tensor $G_{\mu v}=R_{\mu \nu}-\frac{1}{2} \eta_{\mu \nu} R$ with $\eta_{\mu v}=\operatorname{diag}(-1,1,1,1)$ in the orthogonal frame [33] is the following.

$$
\begin{aligned}
G_{00} & =G_{11}=e^{2 \psi-2 \gamma}\left[\frac{1}{\rho} \frac{\partial \gamma}{\partial \rho}-\left(\frac{\partial \psi}{\partial \rho}\right)^{2}-\left(\frac{\partial \psi}{\partial t}\right)^{2}\right], \\
G_{01} & =G_{10}=e^{2 \psi-2 \gamma}\left(\frac{1}{\rho} \frac{\partial \gamma}{\partial t}-2 \frac{\partial \psi}{\partial t} \frac{\partial \psi}{\partial \rho}\right) \\
G_{22} & =e^{2 \psi-2 \gamma}\left[\left(\frac{\partial \psi}{\partial \rho}\right)^{2}-\left(\frac{\partial \psi}{\partial t}\right)^{2}+\frac{\partial^{2} \gamma}{\partial \rho^{2}}-\frac{\partial^{2} \gamma}{\partial t^{2}}\right], \\
G_{33} & =e^{2 \psi-2 \gamma}\left[\left(\frac{\partial \psi}{\partial \rho}\right)^{2}-\left(\frac{\partial \psi}{\partial t}\right)^{2}+\frac{\partial^{2} \gamma}{\partial \rho^{2}}-\frac{\partial^{2} \gamma}{\partial t^{2}}\right] \\
& +2 e^{2 \psi-2 \gamma}\left[\frac{\partial^{2} \psi}{\partial t^{2}}-\frac{\partial^{2} \psi}{\partial \rho^{2}}-\frac{1}{\rho} \frac{\partial \psi}{\partial \rho}\right] .
\end{aligned}
$$

We consider the gravitational field outside the source, i.e., $\rho \neq 0$, which satisfies $G_{\mu \nu}=0$. For $G_{\mu \nu}=0$, Equations (2)-(5) can be simplified as [32] follows.

$$
\begin{aligned}
& \frac{\partial \gamma}{\partial \rho}=\rho\left(\frac{\partial \psi}{\partial t}\right)^{2}+\rho\left(\frac{\partial \psi}{\partial \rho}\right)^{2}, \\
& \frac{\partial \gamma}{\partial t}=2 \rho \frac{\partial \psi}{\partial t} \frac{\partial \psi}{\partial \rho}
\end{aligned}
$$

For gravitational wave solutions, we require $\frac{\partial \psi}{\partial t} \neq 0$. Then, we obtain the equation of $\psi$ from Equations (6) and (7) [32]

$$
-\frac{\partial^{2} \psi}{\partial t^{2}}+\frac{\partial^{2} \psi}{\partial \rho^{2}}+\frac{1}{\rho} \frac{\partial \psi}{\partial \rho}=0
$$

What is noteworthy is that Equation (8) can be obtain more directly from Equations (4) and (5). The equation of $\psi(8)$ is a linear equation. The general solution of Equation (8) is the following:

$$
\psi=\int_{0}^{\infty} d \lambda A_{\lambda} J_{0}(\lambda \rho) \cos \left(\lambda t+\alpha_{\lambda}\right)+\int_{0}^{\infty} d \lambda B_{\lambda} Y_{0}(\lambda \rho) \cos \left(\lambda t+\beta_{\lambda}\right)+\kappa_{1} t+\kappa_{2} \ln \rho+\kappa_{0},
$$

where $\alpha_{\lambda}, \beta_{\lambda}, A_{\lambda}$, and $B_{\lambda}$ are arbitrary functions of $\lambda, J_{0}$ is the Bessel function of first kind, $Y_{0}$ is the Bessel function of second kind, and $\kappa_{0}, \kappa_{1}$, and $\kappa_{2}$ are arbitrary constants. Equations of $\gamma(6)$ and (7) are also linear equations. Substituting Equation (9) into Equations (6) and (7) provides the general solution of $\gamma$ as follows:

$$
\begin{gathered}
\gamma=2 \kappa_{1} \kappa_{2} t+\frac{1}{2} \kappa_{1}^{2} \rho^{2}+\kappa_{2}^{2} \ln \rho+\kappa_{3}-\frac{\rho}{2} \int_{0}^{\infty} d \lambda_{1} \int_{0}^{\infty} d \lambda_{2}\left(F_{+}+F_{-}\right) \\
-2 \kappa_{1} \rho \int_{0}^{\infty} d \lambda\left[A_{\lambda} J_{1}(\lambda \rho) \sin \left(\lambda t+\alpha_{\lambda}\right)+B_{\lambda} Y_{1}(\lambda \rho) \sin \left(\lambda t+\beta_{\lambda}\right)\right] \\
-2 \kappa_{2} \int_{0}^{\infty} d \lambda\left[A_{\lambda} J_{1}(\lambda \rho) \cos \left(\lambda t+\alpha_{\lambda}\right)+B_{\lambda} Y_{1}(\lambda \rho) \cos \left(\lambda t+\beta_{\lambda}\right)\right]
\end{gathered}
$$

with

$$
\begin{aligned}
F_{+} & =\frac{\lambda_{1} \lambda_{2}}{\lambda_{1}+\lambda_{2}}\left\{A_{\lambda_{1}} A_{\lambda_{2}}\left[J_{0}\left(\lambda_{1} \rho\right) J_{1}\left(\lambda_{2} \rho\right)+J_{0}\left(\lambda_{2} \rho\right) J_{1}\left(\lambda_{1} \rho\right)\right] \cos \left(\lambda_{1} t+\lambda_{2} t+\alpha_{\lambda_{1}}+\alpha_{\lambda_{2}}\right)\right. \\
& +A_{\lambda_{1}} B_{\lambda_{2}}\left[J_{0}\left(\lambda_{1} \rho\right) Y_{1}\left(\lambda_{2} \rho\right)+Y_{0}\left(\lambda_{2} \rho\right) J_{1}\left(\lambda_{1} \rho\right)\right] \cos \left(\lambda_{1} t+\lambda_{2} t+\alpha_{\lambda_{1}}+\beta_{\lambda_{2}}\right) \\
& +B_{\lambda_{1}} A_{\lambda_{2}}\left[Y_{0}\left(\lambda_{1} \rho\right) J_{1}\left(\lambda_{2} \rho\right)+J_{0}\left(\lambda_{2} \rho\right) Y_{1}\left(\lambda_{1} \rho\right)\right] \cos \left(\lambda_{1} t+\lambda_{2} t+\beta_{\lambda_{1}}+\alpha_{\lambda_{2}}\right) \\
& \left.+B_{\lambda_{1}} B_{\lambda_{2}}\left[Y_{0}\left(\lambda_{1} \rho\right) Y_{1}\left(\lambda_{2} \rho\right)+Y_{0}\left(\lambda_{2} \rho\right) Y_{1}\left(\lambda_{1} \rho\right)\right] \cos \left(\lambda_{1} t+\lambda_{2} t+\beta_{\lambda_{1}}+\beta_{\lambda_{2}}\right)\right\}
\end{aligned}
$$


and also the following.

$$
\begin{aligned}
F_{-} & =\frac{\lambda_{1} \lambda_{2}}{\lambda_{1}-\lambda_{2}}\left\{A_{\lambda_{1}} A_{\lambda_{2}}\left[J_{0}\left(\lambda_{1} \rho\right) J_{1}\left(\lambda_{2} \rho\right)-J_{0}\left(\lambda_{2} \rho\right) J_{1}\left(\lambda_{1} \rho\right) \cos \left(\lambda_{1} t-\lambda_{2} t+\alpha_{\lambda_{1}}-\alpha_{\lambda_{2}}\right)\right]\right. \\
& +A_{\lambda_{1}} B_{\lambda_{2}}\left[J_{0}\left(\lambda_{1} \rho\right) Y_{1}\left(\lambda_{2} \rho\right)-Y_{0}\left(\lambda_{2} \rho\right) J_{1}\left(\lambda_{1} \rho\right)\right] \cos \left(\lambda_{1} t-\lambda_{2} t+\alpha_{\lambda_{1}}-\beta_{\lambda_{2}}\right) \\
& +B_{\lambda_{1}} A_{\lambda_{2}}\left[Y_{0}\left(\lambda_{1} \rho\right) J_{1}\left(\lambda_{2} \rho\right)-J_{0}\left(\lambda_{2} \rho\right) Y_{1}\left(\lambda_{1} \rho\right)\right] \cos \left(\lambda_{1} t-\lambda_{2} t+\beta_{\lambda_{1}}-\alpha_{\lambda_{2}}\right) \\
& \left.+B_{\lambda_{1}} B_{\lambda_{2}}\left[Y_{0}\left(\lambda_{1} \rho\right) Y_{1}\left(\lambda_{2} \rho\right)-Y_{0}\left(\lambda_{2} \rho\right) Y_{1}\left(\lambda_{1} \rho\right)\right] \cos \left(\lambda_{1} t-\lambda_{2} t+\beta_{\lambda_{1}}-\beta_{\lambda_{2}}\right)\right\} .
\end{aligned}
$$

Here $\kappa_{3}$ in Equation (10) can be eliminated by a coordinate transformation and, without loss of generality, we set $\kappa_{3}=0$.

The solutions (9) and (10) are the general solution of the cylindrical gravitational wave. Other solutions given in Reference [32] and solutions mentioned below are particular cases of the solutions (9) and (10).

\section{Source in Gravitational Wave}

In this section, we show that some gravitational waves possess a source while others do not.

By taking the following:

$$
\begin{aligned}
& B_{\lambda}=B \delta(\omega-\lambda), \\
& A_{\lambda}=\kappa_{0}=\kappa_{1}=\kappa_{2}=0
\end{aligned}
$$

in Equations (9) and (10), where $\omega \neq 0$ is a constant and $\alpha, \beta$ are arbitrary constants, we obtain the following particular solution:

$$
\begin{aligned}
\psi_{\mathrm{s}} & =B Y_{0}(\omega \rho) \cos (\omega t+\beta), \\
\gamma_{\mathrm{s}} & =\frac{B^{2}}{4} \omega^{2} \rho^{2}\left[Y_{0}^{2}(\omega \rho)+2 Y_{1}^{2}(\omega \rho)-Y_{0}(\omega \rho) Y_{2}(\omega \rho)\right] \\
& -\frac{B^{2}}{2} \omega \rho Y_{0}(\omega \rho) Y_{1}(\omega \rho) \cos (2 \omega t+2 \beta)
\end{aligned}
$$

with $\alpha=\alpha_{\omega}$ and $\beta=\beta_{\omega}$.

By taking the following:

$$
\begin{aligned}
& A_{\lambda}=A \delta(\omega-\lambda), \\
& B_{\lambda}=\kappa_{0}=\kappa_{1}=\kappa_{2}=0
\end{aligned}
$$

in Equations (9) and (10), where $\omega \neq 0$ is a constant and $\alpha, \beta$ are arbitrary constants, we obtain another particular solution:

$$
\begin{aligned}
\psi_{\mathrm{sf}} & =A J_{0}(\omega \rho) \cos (\omega t+\alpha), \\
\gamma_{\mathrm{sf}} & =\frac{A^{2}}{4} \omega^{2} \rho^{2}\left[J_{0}^{2}(\omega \rho)+2 J_{1}^{2}(\omega \rho)-J_{0}(\omega \rho) J_{2}(\omega \rho)\right] \\
& -\frac{A^{2}}{2} \omega \rho J_{0}(\omega \rho) J_{1}(\omega \rho) \cos (2 \omega t+2 \alpha),
\end{aligned}
$$

with $\alpha=\alpha_{\omega}$ and $\beta=\beta_{\omega}$.

These two solutions, $\psi_{\mathrm{s}}\left(\gamma_{\mathrm{s}}\right)$ and $\psi_{\mathrm{sf}}\left(\gamma_{\mathrm{sf}}\right)$, have been provided as gravitational wave solutions in Reference [32]. Here, the subscript "s" means "source" and the subscript "sf" means "source-free". We remind the reader that $\psi_{\mathrm{s}}\left(\gamma_{\mathrm{s}}\right)$ and $\psi_{\mathrm{sf}}\left(\gamma_{\mathrm{sf}}\right)$ cannot be expressed as static solutions with coordinates transformations. More details about the static solution can be found in Reference [6].

Gravitational waves solutions $\psi_{\mathrm{s}}\left(\gamma_{\mathrm{s}}\right)$ and $\psi_{\mathrm{sf}}\left(\gamma_{\mathrm{sf}}\right)$ are vacuum solutions for $\rho>0$. Nevertheless, they may possess a source along the $z$ axis at $\rho=0$ [32]. In this paper, we show that the solution $\psi_{\mathrm{s}}\left(\gamma_{\mathrm{s}}\right)$ possesses a source while the solution $\psi_{\mathrm{sf}}\left(\gamma_{\mathrm{sf}}\right)$ does not. 
The above solutions are vacuum solutions for $\rho>0$ and sources can only locate at $\rho=0$. We take the electrostatic field as an example to illustrate the viewpoint. By solving the following source free electrostatic field equation:

$$
-\nabla^{2} \phi=0
$$

we obtain the following solution.

$$
\phi_{1}=\frac{Q}{r} .
$$

However, the solution only makes sense for $r>0$. The solution is singular at $r=0$. By analyzing the singularity at $r=0$, we will find the following.

$$
-\nabla^{2} \phi_{1}=4 \pi Q \delta(\mathbf{r})
$$

That is, there is a Dirac delta source (charge) $Q$ at the singularity $r=0$ [34]. In Reference [35], we show that there is a Dirac delta source at $r=0$ in the Schwarzschild spacetime.

We use the standard mathematical analysis to calculate the energy-momentum tensor at $\rho=0$. More details of this method can be found in our previous work [35]. By replacing $\rho$ by $\sqrt{\rho^{2}+\epsilon^{2}}$ in Equations (12) and (13) and substituting the metric (1) into Equations (2)-(5) by the Einstein equation $G_{\mu v}=8 \pi T_{\mu v}$, we obtain the following.

$$
\begin{aligned}
T_{00}(\epsilon) & =T_{11}(\epsilon)=e^{2 \psi-2 \gamma} \frac{B^{2}}{8 \pi} \frac{\omega^{2} \epsilon^{2}}{\rho^{2}+\epsilon^{2}} Y_{1}^{2}\left(\omega \sqrt{\rho^{2}+\epsilon^{2}}\right) \cos ^{2}(\omega t+\beta), \\
T_{01}(\epsilon) & =e^{2 \psi-2 \gamma} \frac{B^{2}}{8 \pi} \frac{\omega^{2} \epsilon^{2}}{\rho \sqrt{\rho^{2}+\epsilon^{2}}} Y_{0}\left(\omega \sqrt{\rho^{2}+\epsilon^{2}}\right) Y_{1}\left(\omega \sqrt{\rho^{2}+\epsilon^{2}}\right) \sin (2 \omega t+2 \beta), \\
T_{22}(\epsilon) & =e^{2 \psi-2 \gamma} \frac{B^{2}}{8 \pi} \frac{\omega^{2} \epsilon^{2}}{\rho^{2}+\epsilon^{2}} Y_{1}^{2}\left(\omega \sqrt{\rho^{2}+\epsilon^{2}}\right) \cos ^{2}(\omega t+\beta) \\
& -e^{2 \psi-2 \gamma} \frac{B^{2}}{8 \pi} \frac{2 \omega^{3} \epsilon^{2}}{\sqrt{\rho^{2}+\epsilon^{2}}} Y_{0}\left(\omega \sqrt{\rho^{2}+\epsilon^{2}}\right) Y_{1}\left(\omega \sqrt{\rho^{2}+\epsilon^{2}}\right) \cos (2 \omega t+2 \beta) \\
T_{33}(\epsilon) & =e^{2 \psi-2 \gamma} \frac{B^{2}}{8 \pi} \frac{\omega^{2} \epsilon^{2}}{\rho^{2}+\epsilon^{2}} Y_{1}^{2}\left(\omega \sqrt{\rho^{2}+\epsilon^{2}}\right) \cos ^{2}(\omega t+\beta) \\
& -e^{2 \psi-2 \gamma} \frac{B^{2}}{8 \pi} \frac{2 \omega^{3} \epsilon^{2}}{\sqrt{\rho^{2}+\epsilon^{2}}} Y_{0}\left(\omega \sqrt{\rho^{2}+\epsilon^{2}}\right) Y_{1}\left(\omega \sqrt{\rho^{2}+\epsilon^{2}}\right) \cos (2 \omega t+2 \beta) \\
& +e^{2 \psi-2 \gamma} \frac{B^{2}}{8 \pi} \frac{2 \omega^{2} \epsilon^{2}}{\rho^{2}+\epsilon^{2}} Y_{2}\left(\omega \sqrt{\rho^{2}+\epsilon^{2}}\right) \cos (\omega t+\beta) .
\end{aligned}
$$

The energy-momentum tensor is given by the following.

$$
T_{\mu v}=\lim _{\epsilon \rightarrow 0} T_{\mu \nu}(\epsilon) .
$$

When $\rho \neq 0$, we have the following.

$$
T_{\mu \nu}=\lim _{\epsilon \rightarrow 0} T_{\mu v}(\epsilon)=0 .
$$

When $\rho=0$, with the following:

$$
\begin{aligned}
& \lim _{z \rightarrow 0} Y_{0}(z)=\frac{2}{\pi} \ln z+\frac{2}{\pi} \gamma, \\
& \lim _{z \rightarrow 0} Y_{v}(z)=-\frac{\Gamma(v)}{\pi}\left(\frac{z}{2}\right)^{-v}, v \neq 0,
\end{aligned}
$$


where $\gamma$ is the Euler constant, we obtain the following.

$$
\begin{aligned}
& T_{00}=T_{11}=e^{2 \psi-2 \gamma} \frac{B^{2}}{2 \pi^{3}} \cos ^{2}(\omega t+\beta) \lim _{\epsilon \rightarrow 0} \frac{\epsilon^{2}}{\left(\rho^{2}+\epsilon^{2}\right)^{2}}, \\
& T_{01}=-e^{2 \psi-2 \gamma} \frac{B^{2}}{2 \pi^{3}} \sin (2 \omega t+2 \beta) \lim _{\epsilon \rightarrow 0} \frac{\omega \epsilon^{2}}{\rho\left(\rho^{2}+\epsilon^{2}\right)} \ln \left(\omega \sqrt{\rho^{2}+\epsilon^{2}}\right), \\
& T_{22}=e^{2 \psi-2 \gamma} \frac{B^{2}}{2 \pi^{3}} \cos ^{2}(\omega t+\beta) \lim _{\epsilon \rightarrow 0} \frac{\epsilon^{2}}{\left(\rho^{2}+\epsilon^{2}\right)^{2}}, \\
& T_{33}=e^{2 \psi-2 \gamma} \frac{B^{2}}{2 \pi^{3}}\left[\cos ^{2}(\omega t+\beta)-\frac{2 \pi}{B} \cos (\omega t+\beta)\right] \lim _{\epsilon \rightarrow 0} \frac{\epsilon^{2}}{\left(\rho^{2}+\epsilon^{2}\right)^{2}} .
\end{aligned}
$$

By use of [36]:

$$
\lim _{\epsilon \rightarrow 0} \frac{\epsilon^{2}}{\left(x^{2}+y^{2}+\epsilon^{2}\right)^{2}}=\pi \delta(x) \delta(y),
$$

we have the following.

$$
\begin{aligned}
& T_{00}=T_{11}=e^{2 \psi-2 \gamma} \frac{B^{2}}{2 \pi^{2}} \delta(x) \delta(y) \cos ^{2}(\omega t+\beta), \\
& T_{22}=e^{2 \psi-2 \gamma} \frac{B^{2}}{2 \pi^{2}} \delta(x) \delta(y) \cos ^{2}(\omega t+\beta), \\
& T_{33}=e^{2 \psi-2 \gamma} \frac{B^{2}}{2 \pi^{2}} \delta(x) \delta(y)\left[\cos ^{2}(\omega t+\beta)-\frac{2 \pi}{B} \cos (\omega t+\beta)\right] .
\end{aligned}
$$

This is due to the following.

$$
\lim _{\epsilon \rightarrow 0} \frac{T_{01}(\omega, \epsilon)}{T_{00}(\omega, \epsilon)}=0,
$$

$T_{01}(\omega)$ should be regraded as zero.

$$
T_{01}(\omega)=0 .
$$

We show $T_{01}(\omega)=0$ with another method in the Appendix A.

The energy-momentum tensor in the solution $\psi_{\mathrm{s}}\left(\gamma_{\mathrm{s}}\right)$ is then shown as follows:

$$
\begin{aligned}
T_{\mu \nu} & =e^{2 \psi-2 \gamma} \frac{B^{2}}{2 \pi^{2}} \delta(x) \delta(y) \operatorname{diag}\left(\cos ^{2}(\omega t+\beta),\right. \\
& \left.\cos ^{2}(\omega t+\beta), \cos ^{2}(\omega t+\beta), \cos ^{2}(\omega t+\beta)-\frac{2 \pi}{B} \cos (\omega t+\beta)\right)
\end{aligned}
$$

with $x=\rho \cos \phi$ and $y=\rho \sin \phi$. Equation (32) is a time-varying source. That is, the gravitational wave solution $\psi_{\mathrm{s}}\left(\gamma_{\mathrm{s}}\right)$ possesses a source (32) at $\rho=0$.

Replacing $\rho$ by $\sqrt{\rho^{2}+\epsilon^{2}}$ in Equations (15) and (16) and substituting the metric (1) into Equations (2)-(5) by the Einstein equation $G_{\mu \nu}=8 \pi T_{\mu \nu}$, we obtain the following. 


$$
\begin{aligned}
T_{00}(\epsilon) & =T_{11}(\epsilon)=e^{2 \psi-2 \gamma} \frac{A^{2}}{8 \pi} \frac{\omega^{2} \epsilon^{2}}{\rho^{2}+\epsilon^{2}} J_{1}^{2}\left(\omega \sqrt{\rho^{2}+\epsilon^{2}}\right) \cos ^{2}(\omega t+\alpha), \\
T_{01}(\epsilon) & =e^{2 \psi-2 \gamma} \frac{A^{2}}{8 \pi} \frac{\omega^{2} \epsilon^{2}}{\rho \sqrt{\rho^{2}+\epsilon^{2}}} J_{0}\left(\omega \sqrt{\rho^{2}+\epsilon^{2}}\right) J_{1}\left(\omega \sqrt{\rho^{2}+\epsilon^{2}}\right) \sin (2 \omega t+2 \alpha), \\
T_{22}(\epsilon) & =e^{2 \psi-2 \gamma} \frac{A^{2}}{8 \pi} \frac{\omega^{2} \epsilon^{2}}{\rho^{2}+\epsilon^{2}} J_{1}^{2}\left(\omega \sqrt{\rho^{2}+\epsilon^{2}}\right) \cos ^{2}(\omega t+\alpha) \\
& -e^{2 \psi-2 \gamma} \frac{A^{2}}{8 \pi} \frac{2 \omega^{3} \epsilon^{2}}{\sqrt{\rho^{2}+\epsilon^{2}}} J_{0}\left(\omega \sqrt{\rho^{2}+\epsilon^{2}}\right) J_{1}\left(\omega \sqrt{\rho^{2}+\epsilon^{2}}\right) \cos (2 \omega t+2 \alpha), \\
T_{33}(\epsilon) & =e^{2 \psi-2 \gamma} \frac{A^{2}}{8 \pi} \frac{\omega^{2} \epsilon^{2}}{\rho^{2}+\epsilon^{2}} J_{1}^{2}\left(\omega \sqrt{\rho^{2}+\epsilon^{2}}\right) \cos ^{2}(\omega t+\alpha) \\
& -e^{2 \psi-2 \gamma} \frac{A^{2}}{8 \pi} \frac{2 \omega^{3} \epsilon^{2}}{\sqrt{\rho^{2}+\epsilon^{2}}} J_{0}\left(\omega \sqrt{\rho^{2}+\epsilon^{2}}\right) J_{1}\left(\omega \sqrt{\rho^{2}+\epsilon^{2}}\right) \cos (2 \omega t+2 \alpha) \\
& +e^{2 \psi-2 \gamma} \frac{A}{8 \pi} \frac{2 \omega^{2} \epsilon^{2}}{\rho^{2}+\epsilon^{2}} J_{2}\left(\omega \sqrt{\rho^{2}+\epsilon^{2}}\right) \cos (\omega t+\alpha) .
\end{aligned}
$$

When $\rho \neq 0$, we have what follows.

$$
T_{\mu \nu}(\omega)=\lim _{\epsilon \rightarrow 0} T_{\mu \nu}(\omega, \epsilon)=0 .
$$

When $\rho=0$, with the following:

$$
\begin{aligned}
& \lim _{z \rightarrow 0} J_{0}(z)=1, \\
& \lim _{z \rightarrow 0} J_{v}(z)=\frac{1}{\Gamma(v+1)}\left(\frac{z}{2}\right)^{v}, v \neq 0,
\end{aligned}
$$

we obtain what is shown below.

$$
T_{\mu \nu}(\omega)=\lim _{\epsilon \rightarrow 0} T_{\mu \nu}(\omega, \epsilon)=0 .
$$

The solution $\psi_{\mathrm{sf}}\left(\gamma_{\mathrm{sf}}\right)$ is a source free solution. $\psi_{\mathrm{sf}}\left(\gamma_{\mathrm{sf}}\right)$ is source free such that the amplitude can only be determined by the initial conditions.

Actually, one can analyze the Riemann curvature of spacetime described by $\psi_{\text {sf }}\left(\gamma_{\mathrm{sf}}\right)$ and $\psi_{\mathrm{s}}\left(\gamma_{\mathrm{s}}\right)$. In the spacetime described by $\psi_{\mathrm{s}}\left(\gamma_{\mathrm{s}}\right)$, the Riemann curvature blows up at $\rho=0$. In the spacetime described by $\psi_{\text {sf }}\left(\gamma_{\mathrm{sf}}\right)$, the Riemann curvature is finite at $\rho=0$. The singularity of the Riemann curvature implies that the gravitational wave solution possess has a singular source.

\section{Resonance of Gravitational Wave}

In this section, we recognize a resonance in the gravitational wave. A resonance is a particular singular property of vibration.

By taking the following into account:

$$
\begin{aligned}
A_{\lambda} & =A \delta\left(\omega_{1}-\lambda\right), \\
B_{\lambda} & =B \delta\left(\omega_{2}-\lambda\right), \\
\kappa_{1} & =\kappa_{2}=\kappa_{3}=0
\end{aligned}
$$

in the general solutions (9) and (10), we obtain a particular solution: 


$$
\begin{aligned}
\psi_{\mathrm{re}}^{(1)} & =A J_{0}\left(\omega_{1} \rho\right) \cos \left(\omega_{1} t+\alpha_{1}\right)+B Y_{0}\left(\omega_{2} \rho\right) \cos \left(\omega_{2} t+\beta_{2}\right) \\
\gamma_{\mathrm{re}}^{(1)} & =-\frac{A^{2}}{2} \omega_{1} \rho J_{0}\left(\omega_{1} \rho\right) J_{1}\left(\omega_{1} \rho\right) \cos \left(2 \omega_{1} t+2 \alpha_{2}\right)-\frac{B^{2}}{2} \omega_{2} \rho Y_{0}\left(\omega_{2} \rho\right) Y_{1}\left(\omega_{2} \rho\right) \cos \left(2 \omega_{2} t+2 \beta_{2}\right) \\
& +\frac{A^{2}}{4} \omega_{1}^{2} \rho^{2}\left[J_{0}^{2}\left(\omega_{1} \rho\right)+2 J_{1}^{2}\left(\omega_{1} \rho\right)-J_{0}\left(\omega_{1} \rho\right) J_{2}\left(\omega_{1} \rho\right)\right] \\
& +\frac{B^{2}}{4} \omega_{2}^{2} \rho^{2}\left[Y_{0}^{2}\left(\omega_{2} \rho\right)+2 Y_{1}^{2}\left(\omega_{2} \rho\right)-Y_{0}\left(\omega_{2} \rho\right) Y_{2}\left(\omega_{2} \rho\right)\right] \\
& -A B \frac{\omega_{1} \omega_{2}}{\omega_{1}+\omega_{2}} \rho\left[J_{0}\left(\omega_{1} \rho\right) Y_{1}\left(\omega_{2} \rho\right)+Y_{0}\left(\omega_{2} \rho\right) J_{1}\left(\omega_{1} \rho\right)\right] \cos \left(\omega_{1} t+\omega_{2} t+\alpha_{2}+\beta_{2}\right) \\
& -A B \frac{\omega_{1} \omega_{2}}{\omega_{1}-\omega_{2}} \rho\left[J_{0}\left(\omega_{1} \rho\right) Y_{1}\left(\omega_{2} \rho\right)-Y_{0}\left(\omega_{2} \rho\right) J_{1}\left(\omega_{1} \rho\right)\right] \cos \left(\omega_{1} t-\omega_{2} t+\alpha_{1}-\beta_{2}\right)
\end{aligned}
$$

where $\alpha_{1}=\alpha_{\omega_{1}}$ and $\beta_{2}=\beta_{\omega_{2}}$ are arbitrary constants. The subscript "re" means "resonance".

The resonance occurs when $\omega_{1}=\omega_{2}$. When $\omega_{1} \rightarrow \omega_{2}, \gamma_{\text {re }}^{(1)}$ diverges due to the existence of the factor $\frac{1}{\omega_{1}-\omega_{2}}$, this is the typical behavior of a resonance. Taking $\epsilon=$ $\omega_{1}-\omega_{2} \rightarrow 0$, we have the following.

$$
\lim _{\omega_{2} \rightarrow \omega_{1}} \gamma_{\mathrm{re}}^{(1)}=\lim _{\epsilon \rightarrow 0} \gamma_{\mathrm{re}}^{(1)} \sim-\frac{2 A B}{\pi} \omega_{1} \cos \left(\alpha_{1}-\beta_{2}\right) \lim _{\epsilon \rightarrow 0} \frac{1}{\epsilon} .
$$

The resonance effect can be recognized in another method. Taking $\omega_{1}=\omega_{2}=\omega$ directly in what follows:

$$
\begin{aligned}
A_{\lambda} & =A \delta\left(\omega_{1}-\lambda\right), \\
B_{\lambda} & =B \delta\left(\omega_{1}-\lambda\right), \\
\kappa_{1} & =\kappa_{2}=\kappa_{3}=0
\end{aligned}
$$

and substituting them into the general solutions (9) and (10), we obtain the following solution:

$$
\begin{aligned}
\psi_{\mathrm{re}}^{(2)} & =A J_{0}(\omega \rho) \cos (\omega t+\alpha)+B Y_{0}(\omega \rho) \cos (\omega t+\beta), \\
\gamma_{\mathrm{re}}^{(2)} & =f(\rho)-\frac{2 A B}{\pi} \omega t \sin (\alpha-\beta)-\frac{A^{2}}{2} \omega \rho J_{0}(\omega \rho) J_{1}(\omega \rho) \cos (2 \omega t+2 \alpha) \\
& -\frac{B^{2}}{2} \omega \rho Y_{0}(\omega \rho) Y_{1}(\omega \rho) \cos (2 \omega t+2 \beta) \\
& -\frac{A B}{2} \omega \rho\left[J_{1}(\omega \rho) Y_{0}(\omega \rho)+J_{0}(\omega \rho) Y_{1}(\omega \rho)\right] \cos (2 \omega t+\alpha+\beta)
\end{aligned}
$$

with $\alpha=\alpha_{\omega}, \beta=\beta_{\omega}$ and the following is the case.

$$
\begin{aligned}
f(\rho) & =\frac{A^{2}}{4} \omega^{2} \rho^{2}\left[J_{0}^{2}(\omega \rho)+2 J_{1}^{2}(\omega \rho)-J_{0}(\omega \rho) J_{2}(\omega \rho)\right] \\
& +\frac{B^{2}}{4} \omega^{2} \rho^{2}\left[Y_{0}^{2}(\omega \rho)+2 Y_{1}^{2}(\omega \rho)-Y_{0}(\omega \rho) Y_{2}(\omega \rho)\right] \\
& +\frac{A B}{4} \omega^{2} \rho^{2} \cos (\alpha-\beta) \\
& \times\left[2 J_{0}(\omega \rho) Y_{0}(\omega \rho)+4 J_{1}(\omega \rho) Y_{1}(\omega \rho)-J_{0}(\omega \rho) Y_{2}(\omega \rho)-J_{2}(\omega \rho) Y_{0}(\omega \rho)\right] .
\end{aligned}
$$

When $A \neq 0$ and $B \neq 0, \gamma$ in Equation (4) has an aperiodic term proportional to $t$. $\gamma_{\mathrm{re}}^{(2)}$ diverges as $t$ proceeds to infinity. This is also a typical behavior of a resonance, which means the impact of the external force stores up and increases with time $t$. The resonance is indicated by a divergence in $\gamma_{\mathrm{re}}$. When we solve $\psi_{\mathrm{re}}\left(\gamma_{\mathrm{re}}\right)$ with different methods, the divergence appears in different ways. $\gamma_{\mathrm{re}}^{(1)}$ and $\gamma_{\mathrm{re}}^{(2)}$ are just two different normalization 
methods when dealing with a divergence. We can solve a forced harmonic oscillator $\frac{d^{2}}{d t^{2}} x(t)+\omega_{0}^{2} x(t)=\sin \left(\omega_{0} t\right)$ directly or with the Green function method to find the divergence as $t \rightarrow \infty$ or $\lim _{\omega \rightarrow \omega_{0}} \frac{1}{\omega-\omega_{0}}$.

Here, we provide a physical picture of the resonance above. $\psi_{\mathrm{re}}^{(1)}(40)$ or $\psi_{\mathrm{re}}^{(2)}(44)$ is the combination of $\psi_{\mathrm{s}}(12)$ and $\psi_{\mathrm{sf}}(15)$, which means that gravitational waves $\psi_{\mathrm{s}}\left(\gamma_{\mathrm{s}}\right)$ and $\psi_{\text {sf }}\left(\gamma_{\mathrm{sf}}\right)$ coexist. When $\psi_{\mathrm{s}}\left(\gamma_{\mathrm{s}}\right)$ and $\psi_{\mathrm{sf}}\left(\gamma_{\mathrm{sf}}\right)$ have the same frequency $\omega$, they resonate with one another.

What is noteworthy is that the two gravitational wave solutions with sources do not resonate with one another. Two gravitational wave solutions without sources do not resonate with one another either. A resonance only occurs between a gravitational wave with a source and a gravitational wave without source. The calculation will be shown in the later section. In this sense, the gravitational wave with a source and the gravitational wave without a source shall be considered as two different kinds of gravitational waves.

\section{Interference of Gravitational Wave}

General relativity is a nonlinear theory and, thus, the interference of gravitational waves is nontrivial in principle. In this section, we consider the interference of gravitational waves. The interference term will appear both in the metric and in the source.

\subsection{Interference Term in Metric}

The factor $\psi$ in the metric (1) satisfies a linear Equation (8). Thus, the following:

$$
\psi=\psi_{1}+\psi_{2}
$$

is also a solution when $\psi_{1}$ and $\psi_{2}$ are solutions of Equation (8). For convenience, we take $\psi_{1}$ and $\psi_{2}$ given by Equation (12) as an example.

$$
\begin{aligned}
& \psi_{1}=B_{1} Y_{0}\left(\omega_{1} \rho\right) \cos \left(\omega_{1} t+\beta_{1}\right), \\
& \psi_{2}=B_{2} Y_{0}\left(\omega_{2} \rho\right) \cos \left(\omega_{2} t+\beta_{2}\right) .
\end{aligned}
$$

These two radiations have frequencies $\omega_{1}$ and $\omega_{2}$, respectively. Substituting Equation (47) into Equations (6) and (7), we have the following:

$$
\gamma=\gamma_{1}+\gamma_{2}-\gamma_{\text {int }}
$$

where $\gamma_{1}$ and $\gamma_{2}$ are given by Equation (13) with frequencies $\omega_{1}$ and $\omega_{2}$ :

$$
\begin{aligned}
\gamma_{1} & =\frac{B_{1}^{2}}{4} \omega_{1}^{2} \rho^{2}\left[Y_{0}^{2}\left(\omega_{1} \rho\right)+2 Y_{1}^{2}\left(\omega_{1} \rho\right)-Y_{0}\left(\omega_{1} \rho\right) Y_{2}\left(\omega_{1} \rho\right)\right] \\
& -\frac{B_{1}^{2}}{2} \omega_{1} \rho Y_{0}\left(\omega_{1} \rho\right) Y_{1}\left(\omega_{1} \rho\right) \cos \left(2 \omega_{1} t+2 \beta_{1}\right) \\
\gamma_{2} & =\frac{B_{2}^{2}}{4} \omega_{2}^{2} \rho^{2}\left[Y_{0}^{2}\left(\omega_{2} \rho\right)+2 Y_{1}^{2}\left(\omega_{2} \rho\right)-Y_{0}\left(\omega_{2} \rho\right) Y_{2}\left(\omega_{2} \rho\right)\right] \\
& -\frac{B_{2}^{2}}{2} \omega_{2} \rho Y_{0}\left(\omega_{2} \rho\right) Y_{1}\left(\omega_{2} \rho\right) \cos \left(2 \omega_{2} t+2 \beta_{2}\right)
\end{aligned}
$$

and the following is the case.

$$
\begin{aligned}
\gamma_{\text {int }} & =B_{1} B_{2} \frac{\omega_{1} \omega_{2}}{\omega_{1}+\omega_{2}} \rho\left[Y_{0}\left(\omega_{1} \rho\right) Y_{1}\left(\omega_{2} \rho\right)+Y_{0}\left(\omega_{2} \rho\right) Y_{1}\left(\omega_{1} \rho\right)\right] \cos \left(\omega_{1} t+\omega_{2} t+\beta_{1}+\beta_{2}\right) \\
& +B_{1} B_{2} \frac{\omega_{1} \omega_{2}}{\omega_{1}-\omega_{2}} \rho\left[Y_{0}\left(\omega_{1} \rho\right) Y_{1}\left(\omega_{2} \rho\right)-Y_{0}\left(\omega_{2} \rho\right) Y_{1}\left(\omega_{1} \rho\right)\right] \cos \left(\omega_{1} t-\omega_{2} t+\beta_{1}-\beta_{2}\right) .
\end{aligned}
$$

$\gamma_{\text {int }}$ is the interference term between two gravitational waves. $\psi_{1}\left(\gamma_{1}\right)$ and $\psi_{2}\left(\gamma_{2}\right)$ are two gravitational wave solutions with sources. 
We emphasize that in this case when $\omega_{1}=\omega_{2}=\omega$, then the following is the case:

$$
\begin{aligned}
\gamma_{\text {int }} & =B_{1} B_{2} \omega \rho Y_{0}(\omega \rho) Y(\omega \rho) \cos \left(2 \omega t+\beta_{1}+\beta_{2}\right) \\
& -\frac{B_{1} B_{2}}{2} \omega^{2} \rho^{2}\left[Y_{0}^{2}(\omega \rho)+2 Y_{1}^{2}(\omega \rho)-Y_{0}(\omega \rho) Y_{2}(\omega \rho)\right] \cos \left(\beta_{1}-\beta_{2}\right)
\end{aligned}
$$

and the above does not diverge; that is, two gravitational wave solutions with sources do not resonate with each other. What is noteworthy is that two gravitational wave solutions without sources do not resonate with one another either. We repeat that a resonance only occurs between a gravitational wave with a source and a gravitational wave without source.

\subsection{Interference Term in Energy-Momentum Tensor}

Now we calculate the energy-momentum tensor of the solutions (47) and (49).

By the same procedure in Section 2, we obtain the energy-momentum tensor in the spacetime described by the metric (47) and (49):

$$
\begin{aligned}
& T_{00}\left(\omega_{1}, \omega_{2}\right)=T_{11}\left(\omega_{1}, \omega_{2}\right)=T_{00}\left(\omega_{1}\right)+T_{00}\left(\omega_{2}\right)+T_{\text {int }}\left(\omega_{1}, \omega_{2}\right), \\
& T_{01}\left(\omega_{1}, \omega_{2}\right)=T_{10}\left(\omega_{1}, \omega_{2}\right)=0, \\
& T_{22}\left(\omega_{1}, \omega_{2}\right)=T_{22}\left(\omega_{1}\right)+T_{22}\left(\omega_{2}\right)+T_{\text {int }}\left(\omega_{1}, \omega_{2}\right), \\
& T_{33}\left(\omega_{1}, \omega_{2}\right)=T_{33}\left(\omega_{1}\right)+T_{33}\left(\omega_{2}\right)+T_{\text {int }}\left(\omega_{1}, \omega_{2}\right)
\end{aligned}
$$

with $\psi$ and $\gamma$ given in Equations (47) and (49), $T_{\mu v}\left(\omega_{i}\right)(i=1,2)$ given in Equations (28)-(30):

$$
\begin{aligned}
& T_{00}\left(\omega_{i}\right)=e^{2 \psi-2 \gamma} \frac{B_{i}^{2}}{2 \pi^{2}} \delta(x) \delta(y) \cos ^{2}\left(\omega_{i} t+\beta_{i}\right), \\
& T_{22}\left(\omega_{i}\right)=e^{2 \psi-2 \gamma} \frac{B_{i}^{2}}{2 \pi^{2}} \delta(x) \delta(y) \cos ^{2}\left(\omega_{i} t+\beta_{i}\right), \\
& T_{33}\left(\omega_{i}\right)=e^{2 \psi-2 \gamma} \frac{B_{i}^{2}}{2 \pi^{2}} \delta(x) \delta(y)\left[\cos ^{2}\left(\omega_{i} t+\beta_{i}\right)-\frac{2 \pi}{B_{i}} \cos \left(\omega_{i} t+\beta_{i}\right)\right] .
\end{aligned}
$$

and the interference term of the following is given.

$$
T_{\text {int }}\left(\omega_{1}, \omega_{2}\right)=e^{2 \psi-2 \gamma} \frac{B_{1} B_{2}}{\pi^{2}} \delta(x) \delta(y) \cos \left(\omega_{1} t+\beta_{1}\right) \cos \left(\omega_{2} t+\beta_{2}\right) .
$$

$T_{\mu \nu}\left(\omega_{1}, \omega_{2}\right)$ involves both $\omega_{1}$ and $\omega_{2}$ and can be written in three parts: $T_{\mu \nu}\left(\omega_{1}\right)$, which involves only $\omega_{1} ; T_{\mu \nu}\left(\omega_{2}\right)$, which involves only $\omega_{2} ;$ and $T_{\text {int }}\left(\omega_{1}, \omega_{2}\right)$, which involves both $\omega_{1}$ and $\omega_{2}$. $T_{\text {int }}\left(\omega_{1}, \omega_{2}\right)$ is an interference term due to the superposition of gravitational waves.

Nevertheless, although the interference term $T_{\text {int }}\left(\omega_{1}, \omega_{2}\right)$ exists, the time average of $T_{\text {int }}$ vanishes.

$$
\left\langle T_{\text {int }}\right\rangle=\int_{0}^{\infty} d t T_{\text {int }}\left(\omega_{1}, \omega_{2}\right)=0 .
$$

The behavior of the interference term implies that the result of a long-time measurement should superpose linearly. That is, when the typical time of a detector is larger than the period of a gravitational wave, the source of gravitational waves observed may be linear.

As an analogy, we consider the energy superposition of two plane electromagnetic waves. The electromagnetic energy density can serve as the source of gravitational waves. When two plane electromagnetic waves $E_{1}=A_{1} \cos \left(\omega_{1} t+\alpha\right)$ and $E_{2}=A_{2} \cos \left(\omega_{2} t+\beta\right)$ superpose together, the electric field is the following:

$$
E=E_{1}+E_{2}=A_{1} \cos \left(\omega_{1} t+\alpha\right)+A_{2} \cos \left(\omega_{2} t+\beta\right),
$$


where $E$ is the electric field and $A$ is the amplitude. The energy density of the electric field is the following:

$$
\varepsilon=E^{2}=\left(E_{1}+E_{2}\right)^{2}=\varepsilon_{1}+\varepsilon_{2}+\varepsilon_{\text {int }},
$$

where $\varepsilon_{1}=A_{1}^{2} \cos ^{2}\left(\omega_{1} t+\alpha\right), \varepsilon_{2}=A_{2}^{2} \cos ^{2}\left(\omega_{2} t+\beta\right)$ and the interference term is as follows.

$$
\varepsilon_{\text {int }}=2 A_{1} A_{2} \cos \left(\omega_{1} t+\alpha\right) \cos \left(\omega_{2} t+\beta\right) .
$$

The time average of $\varepsilon_{\text {int }}$ vanishes:

$$
\left\langle\varepsilon_{\text {int }}\right\rangle=\int_{0}^{\infty} d t \varepsilon_{\text {int }}=0 .
$$

The behavior of the interference in the energy-momentum tensor (52) is similar to the interference behavior of the energy density (56) in electromagnetic waves.

\section{Conclusions and Outlook}

Most results about gravitational waves are deduced in a linear approximation, such as the gravitational quadrupole radiation, the resonance between the gravitational wave and the detector, and the linear superposition of two gravitational waves. When we take the strict nonlinear theory-exact wave solutions of the Einstein equation-some new properties of gravitational waves come into sight.

In this paper, we discussed gravitational waves based on the exact cylindrical gravitational wave solutions rather than gravitational wave solutions in the linear approximation. Gravitational waves are vacuum solutions outside singularities. Nevertheless, curvature singularities imply that the gravitational wave solution possesses a source at the singularity. We show that the gravitational wave solution $\psi_{\mathrm{s}}\left(\gamma_{\mathrm{s}}\right)$ possesses a source, while $\psi_{\mathrm{sf}}\left(\gamma_{\mathrm{sf}}\right)$ is source-free. We could make an analogy with electromagnetic waves. The electromagnetic radiation is a special kind of electromagnetic wave produced by the variation of the dipole. Nevertheless, not all electromagnetic waves are electromagnetic radiations. A plane electromagnetic wave, as an exact solution, is not an electromagnetic radiation, and an electromagnetic wave produced by the antenna is an electromagnetic radiation. In this sense, $\psi_{\mathrm{s}}\left(\gamma_{\mathrm{s}}\right)$ is a gravitational radiation wave, while $\psi_{\mathrm{sf}}\left(\gamma_{\mathrm{sf}}\right)$ is a pure gravitational wave. At the very least, they are two kinds of gravitational waves. We recognize a resonance between the gravitational waves. It is worth mentioning that the resonance only occurs between a gravitational wave with a source and a gravitational wave without a source. Two gravitational waves with sources and two gravitational waves without sources do not resonate with one another. The resonance also indicates that the gravitational wave with sources and the gravitational wave without sources are two different kinds of gravitational waves. We suppose that the resonance between gravitational waves is irrelevant to the symmetry of the system. In recent years, gravitational wave detection has produced rapid progress. We expect that the resonance between gravitational waves will be found in the future. We investigated the interference of gravitational waves. The interference appears in both the metric and the energy-momentum tensor. Nevertheless, the time average of the interference term in the energy-momentum tensor vanishes, which indicates that the energy-momentum tensor of two gravitational waves superpose linearly at the time-averaging level.

As mentioned above, a gravitational wave with a source should be regarded as a gravitational radiation. Gravitational radiations will result in the energy loss of the source. With the conservation law of the energy, we may define the energy of the cylindrical gravitational radiation in our framework. We can also consider the resonance between matter waves and gravitational waves based on our previous works on scattering [37-39]. 
Author Contributions: Conceptualization, Y.-Z.C., S.-L.L., Y.-J.C. and W.-S.D.; Writing-review \& editing, Y.-J.C. and W.-S.D. All authors have read and agreed to the published version of the manuscript.

Funding: This work is supported in part by the Nankai Zhide foundation and NSF of China under Grant No. 11575125 and No. 11675119.

Institutional Review Board Statement: Not applicable.

Informed Consent Statement: Not applicable.

Data Availability Statement: All data that support the findings of this study are included within the article.

Acknowledgments: We are very indebted to G. Zeitrauman for his encouragement.

Conflicts of Interest: The authors declare no conflict of interest.

\section{Appendix A}

We show $T_{01}(\omega)=0$ in the solution $\psi_{s}\left(\gamma_{s}\right)$ with another method below.

Integrating $T_{00}(\omega, \epsilon)$ over the entire space of the following:

$$
E(\omega, \epsilon) \equiv \int \sqrt{g} d \rho d \theta d \phi T_{00}(\omega, \epsilon)=\frac{\omega^{2} \epsilon^{2} L_{z}}{8}\left[B^{2}\left(Y_{0}^{2}(\omega \epsilon)+Y_{1}^{2}(\omega \epsilon)\right) \cos ^{2}(\omega t+\beta)\right]
$$

and by taking the limit $\epsilon \rightarrow 0$, we have the following

$$
E(\omega) \equiv \lim _{\epsilon \rightarrow 0} E(\omega, \epsilon)=\frac{B^{2} L_{z}}{2 \pi^{2}} \cos ^{2}(\omega t+\beta)
$$

with $L_{z} \equiv \int_{-\infty}^{\infty} d z$. Again, integrating $T_{01}(\epsilon)$ over the entire space, we obtain:

$$
\begin{aligned}
P_{\rho}(\omega, \epsilon) & =\int \sqrt{g} d \rho d \theta d \phi T_{01}(\omega, \epsilon) \\
& =\frac{\omega^{2} \epsilon^{2} L_{z}}{2}\left\{A^{2}\left[\omega \epsilon J_{0}^{2}(\omega \epsilon)+\omega \epsilon J_{1}^{2}(\omega \epsilon)-J_{0}(\omega \epsilon) J_{1}(\omega \epsilon)\right]\right. \\
& \times \sin (\omega t+\alpha) \cos (\omega t+\alpha) \\
& +B^{2}\left[\omega \epsilon Y_{0}^{2}(\omega \epsilon)+\omega \epsilon Y_{1}^{2}(\omega \epsilon)-Y_{0}(\omega \epsilon) Y_{1}(\omega \epsilon)\right] \\
& \times \sin (\omega t+\beta) \cos (\omega t+\beta) \\
& +A B\left[\omega \epsilon J_{0}(\omega \epsilon) Y_{0}(\omega \epsilon)+\omega \epsilon J_{1}(\omega \epsilon) Y_{1}(\omega \epsilon)-J_{1}(\omega \epsilon) Y_{0}(\omega \epsilon)\right] \\
& \times \cos (\omega t+\alpha) \sin (\omega t+\beta) \\
& +A B\left[\omega \epsilon J_{0}(\omega \epsilon) Y_{0}(\omega \epsilon)+\omega \epsilon J_{1}(\omega \epsilon) Y_{1}(\omega \epsilon)-J_{0}(\omega \epsilon) Y_{1}(\omega \epsilon)\right] \\
& \times \sin (\omega t+\alpha) \cos (\omega t+\beta)\}
\end{aligned}
$$

and taking the limit $\epsilon \rightarrow 0$, we have the following.

$$
P_{\rho}(\omega) \equiv \lim _{\epsilon \rightarrow 0} P_{\rho}(\omega, \epsilon)=\lim _{\epsilon \rightarrow 0} \frac{2 B^{2} L_{z} \omega^{2}}{\pi^{2}} \epsilon \ln \epsilon=0
$$

The integration of $T_{01}$ over the entire space is vanishing; thus, we have $T_{01}(\omega)=0$.

\section{References}

1. Hawking, S.W.; Ellis, G.F.R. The Large Scale Structure of Space-Time; Cambridge University Press: Cambridge, UK, 1973 ; Volume 1.

2. Ohanian, H.C.; Ruffini, R. Gravitation and Spacetime; Cambridge University Press: Cambridge, UK, 2013.

3. Li, W.-D.; Li, S.-L.; Chen, Y.-J.; Chen, Y.-Z.; Dai, W.-S. Gravitational wave scattering theory without large-distance asymptotics. Ann. Phys. 2021, 427, 168424. [CrossRef]

4. Weinberg, S. Gravitation and Cosmology: Principles and Applications of the General Theory of Relativity; Wiley: New York, NY, USA, 1972; Volume 1.

5. Misner, C.W.; Thorne, K.S.; Wheeler, J.A. Gravitation; Princeton University Press: Princeton, NJ, USA, 2017. 
6. Marder, L. Gravitational waves in general relativity I. Cylindrical waves. Proc. R. Soc. Lond. Ser. A Math. Phys. Sci. 1958, 244, 524-537.

7. Marder, L. Gravitational waves in general relativity II. The reflexion of cylindrical waves. Proc. R. Soc. Lond. Ser. A Math. Phys. Sci. 1958, 246, 133-143.

8. Pirani, F. Gravitational waves in general relativity. IV. The gravitational field of a fast-moving particle. Proc. R. Soc. Lond. Ser. A Math. Phys. Sci. 1959, 252, 96-101.

9. Marder, L. Gravitational waves in general relativity V. An exact spherical wave. Proc. R. Soc. Lond. Ser. A Math. Phys. Sci. 1961, 261, 91-96.

10. Marder, L. Gravitational waves in general relativity XI. Cylindrical-spherical waves. Proc. R. Soc. Lond. A Math. Phys. Sci. 1969, 313, 83-96.

11. Marder, L. Gravitational waves in general relativity. XII. Correspondence between toroidal and cylindrical waves. Proc. R. Soc. Lond. A Math. Phys. Sci. 1972, 327, 123-130.

12. Thorne, K.S. Energy of infinitely long, cylindrically symmetric systems in general relativity. Phys. Rev. 1965, 138, B251. [CrossRef]

13. Bini, D.; Geralico, A.; Plastino, W. Cylindrical gravitational waves: C-energy, super-energy and associated dynamical effects. Class. Quantum Gravity 2019, 36, 095012. [CrossRef]

14. Garecki, J. Do gravitational waves carry energy-momentum? A reappraisal. arXiv 2016, arXiv:1612.07077.

15. Dominguez, P.; Gallegos, A.; Macias-Diaz, J.; Vargas-Rodriguez, H. Superenergy flux of Einstein-Rosen waves. Int. J. Mod. Phys. D 2018, 27, 1850072. [CrossRef]

16. Tomizawa, S.; Mishima, T. Nonlinear effects for a cylindrical gravitational two-soliton. Phys. Rev. D 2015, 91, 124058. [CrossRef]

17. Celestino, J.; de Oliveira, H.; Rodrigues, E. Nonlinear evolution of cylindrical gravitational waves: Numerical method and physical aspects. Phys. Rev. D 2016, 93,104018. [CrossRef]

18. Bini, D.; Chicone, C.; Mashhoon, B. Twisted gravitational waves. Phys. Rev. D 2018, 97, 064022. [CrossRef]

19. Chandrasekhar, S.; Ferrari, V. On the dispersion of cylindrical impulsive gravitational waves. Proc. R. Soc. Lond. A Math. Phys. Sci. 1987, 412, 75-91.

20. Chakraborty, S.; Chakraborty, S. Gravitational collapse of dissipative fluid as a source of gravitational waves. Ann. Phys. 2016, 364, 110-119. [CrossRef]

21. Stachel, J.J. Cylindrical gravitational news. J. Math. Phys. 1966, 7, 1321-1331. [CrossRef]

22. Garriga, J.; Verdaguer, E. Cosmic strings and Einstein-Rosen soliton waves. Phys. Rev. D 1987, 36, 2250. [CrossRef] [PubMed]

23. Xanthopoulos, B.C. Cosmic strings coupled with gravitational and electromagnetic waves. Phys. Rev. D 1987, 35, 3713. [CrossRef]

24. Berger, B.K.; Chrusciel, P.; Moncrief, V. On "Asymptotically Flat" Space-Times with G 2-Invariant Cauchy Surfaces. Ann. Phys. 1995, 237, 322-354. [CrossRef]

25. Korotkin, D.; Samtleben, H. Canonical quantization of cylindrical gravitational waves with two polarizations. Phys. Rev. Lett. 1998, 80, 14. [CrossRef]

26. Kuchar, K. Canonical quantization of cylindrical gravitational waves. Phys. Rev. D 1971, 4, 955. [CrossRef]

27. Ashtekar, A.; Pierri, M. Probing quantum gravity through exactly soluble midi-superspaces I. J. Math. Phys. 1996, 37, 6250-6270. [CrossRef]

28. Detweiler, S. Black holes and gravitational waves. III-The resonant frequencies of rotating holes. Astrophys. J. 1980, 239, 292-295. [CrossRef]

29. Hopman, C.; Alexander, T. Resonant relaxation near a massive black hole: The stellar distribution and gravitational wave sources. Astrophys. J. 2006, 645, 1152. [CrossRef]

30. Mizuno, J.; Strain, K.A.; Nelson, P.; Chen, J.; Schilling, R.; Rüdiger, A.; Winkler, W.; Danzmann, K. Resonant sideband extraction: A new configuration for interferometric gravitational wave detectors. Phys. Lett. A 1993, 175, 273-276. [CrossRef]

31. Staley, A.; Martynov, D.; Abbott, R.; Adhikari, R.; Arai, K.; Ballmer, S.; Barsotti, L.; Brooks, A.; DeRosa, R.; Dwyer, S.; et al. Achieving resonance in the Advanced LIGO gravitational-wave interferometer. Class. Quantum Gravity 2014, 31, 245010. [CrossRef]

32. Carmeli, M. Classical Fields: General Relativity and Gauge Theory; World Scientific Publishing Company: Singapore, 2001.

33. Chen, Y.-Z.; Li, W.-D.; Dai, W.-S. A 1+5-dimensional gravitational-wave solution: Curvature singularity and spacetime singularity. Eur. Phys. J. C 2017, 77, 859. [CrossRef]

34. Jackson, J.D. Classical Electrodynamics; American Association of Physics Teachers: College Park, MD, USA, 1999.

35. Chen, Y.-Z.; Dai, W.-S. Singular vacuum solutions as singular matter solutions: Where do spacetime singularities come from? EPL (Europhys. Lett.) 2017, 120, 10004. [CrossRef]

36. Ibragimov, N.H. A Practical Course in Differential Equations and Mathematical Modelling: Classical and New Methods. Nonlinear Mathematical Models. Symmetry and Invariance Principles; World Scientific Publishing Company: Singapore, 2009.

37. Liu, T.; Li, W.-D.; Dai, W.-S. Scattering theory without large-distance asymptotics. J. High Energy Phys. 2014, 2014, 1-12. [CrossRef]

38. Li, W.-D.; Chen, Y.-Z.; Dai, W.-S. Scalar scattering in Schwarzschild spacetime: Integral equation method. Phys. Lett. B 2018, 786, 300-304. [CrossRef]

39. Li, W.-D.; Chen, Y.-Z.; Dai, W.-S. Scattering state and bound state of scalar field in Schwarzschild spacetime: Exact solution. Ann. Phys. 2019, 409, 167919. [CrossRef] 